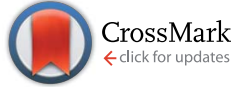

Cite this: RSC Adv., 2016, 6, 84730

Received 30th June 2016

Accepted 2nd September 2016

DOI: $10.1039 / c 6 r a 16889 b$

www.rsc.org/advances

\section{Spiro-condensation of 5-methoxycarbonyl-1H- pyrrole-2,3-diones with cyclic enoles to form spiro substituted furo[3,2-c]-coumarins and quinolines $\uparrow$}

\author{
Alexey Yu. Dubovtsev, ${ }^{a}$ Pavel S. Silaichev, ${ }^{a}$ Mikhail A. Nazarov, ${ }^{a}$ Maksim V. Dmitriev, ${ }^{a}$ \\ Andrey N. Maslivets ${ }^{\star a}$ and Michael Rubin ${ }^{\star b c}$
}

Highly efficient spiro-condensation enabling cyclic enoles to act as 1,3-bis-nucleophiles in reaction with pyrrole-2,3-diones acting as 1,2-bis-electrophiles was developed. The corresponding furo[3,2-c] coumarins and furo[3,2-c]quinolines containing a spiro pyrrole fragment were obtained in high yields.

\section{Introduction}

Natural products containing furo[3,2-c] $\operatorname{coumarin}^{1}$ and furo[3,2$c$ ]quinolone ${ }^{2}$ fragments have been the subject of great interest due to their important biological properties. For example, phyto-alkaloids hypocrolide A, glaumacidine, coumestrol (coumestan), and their synthetic analogs demonstrated promising antimitotic, antiprolifirative and cytotoxic activities (Scheme 1). Not surprisingly, development of new synthetic routes to these scaffolds is of great interest for modern synthetic and medicinal chemistry. Herein we wish to disclose

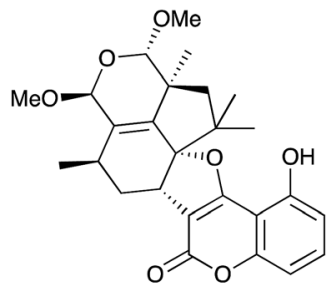

Hypocrolide A

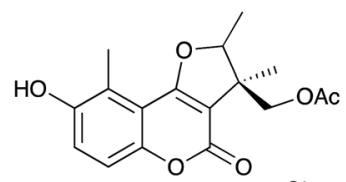

Glaumacidine

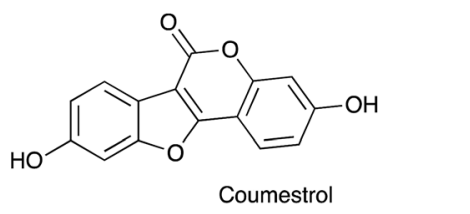

Scheme 1
${ }^{a}$ Department of Chemistry, Perm State University, ul. Bukireva 15, Perm 614990, Russia.E-mail:koh2@psu.ru

${ }^{b}$ Department of Chemistry, North Caucasus Federal University, 1a Pushkin St., Stavropol 355009, Russian Federation

'Department of Chemistry, University of Kansas, 1251 Wescoe Hall Dr., Lawrence, KS 66045-7582, USA. E-mail: mrubin@ku.edu; Fax: +1 785864 5396; Tel: +1 785864 5071

† Electronic supplementary information (ESI) available: Experimental procedures, physico-chemical and spectral data. CCDC 1486439 and 1486440. For ESI and crystallographic data in CIF or other electronic format see DOI: 10.1039/c6ra16889b the results of our synthetic studies towards spiro analogs of furo[3,2-c]coumarins and furo[3,2-c] quinolones. The featured synthetic strategy is based on highly selective electrophilic reactions of $1 H$-pyrrole-2,3-diones.

\section{Results and discussion}

Due to their high reactivity and pronounced electrophilic properties $1 H$-pyrrole-2,3-diones are often employed as useful building blocks allowing for efficient incorporation of a nitrogen containing five-membered heterocyclic unit in the structure of a target molecule. Such approach has found application in total synthesis of natural alkaloids. ${ }^{3}$ The installation of additional electron-withdrawing groups (such as acyl, alkoxycarbonyl or 1,2-dicarbonyl moieties) at C-4 and C-5 of the pyrrole ring may further increase synthetic versatility of these compounds. Diverse reactions of such 4,5-disubstituted $1 H^{-}$ pyrrole-2,3-diones can be used for expeditious assembly of various fused and bridged, polyheterocyclic scaffolds, often hardly available or unavailable by other methods. ${ }^{4}$ Furthermore, the installation of an ester function at C-5 provides an additional electrophilic moiety in multistep cascade reactions with bis-nucleophilic reagents, offering easy access towards functionalized spiro-pyrroles (Scheme 2). Thus, we previously demonstrated the reaction of 5-methoxycarbonyl- $1 H$-pyrrole2,3-diones 1 with 1,3- $N, N$-bis-nuclephiles, such as urea derivatives 2 to yield derivatives of 1,3,6-triazaspiro[4,4]nonane 3 (Scheme 2, path A). ${ }^{5}$ Similarly, utilization of enamines 4 as 1,3$C, N$-bisnucleophiles afforded products with 1,7-diazaspiro[4,4] nonane scaffold 5 (Scheme 2, path B). ${ }^{6,7}$ A detailed investigation of this transformation revealed that both acyclic ${ }^{6}$ and cyclic ${ }^{7}$ enamines can be readily employed as bis-nucleophiles, providing facile access to annulated functionalized spiranes $\mathbf{5}$ with increased molecular complexity. At the same time, to the best of our knowledge, related spiro-condensations of monocyclic $1 H$-pyrrole-2,3-diones 1 with enoles 6 (Scheme 2, path C) is still unknown. We envisioned that such a process, if 


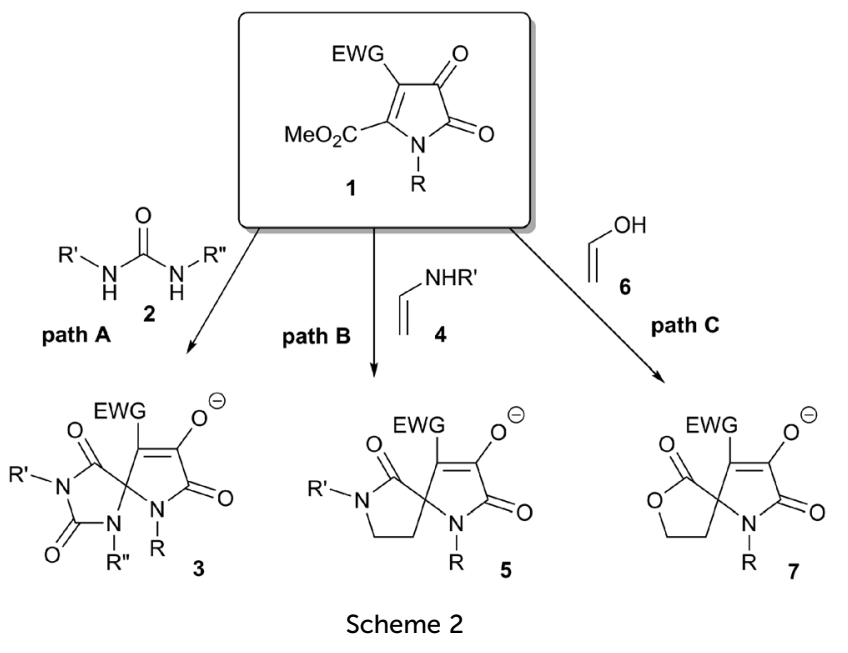

successful, might serve for efficient preparation of 7-oxa-1azaspiro[4,4]nonanes 7 , including spiro-derivatives of furo[3,2$c]$ coumarins and furo[3,2-c] quinolones.

To this end we envisioned that stable enol forms such as 4hydroxycoumarine (6a, $\mathrm{X}=\mathrm{O})$ and 4-hydroxyquinolin-2 $(1 H)$ ones $(\mathbf{6 b}, \mathrm{X}=\mathrm{NMe} ; \mathbf{6 b}, \mathrm{X}=\mathrm{NPh})$ could serve as suitable precursors for the designed transformation. Indeed, enolate $\mathbf{8}$ generated in the presence of catalytic base is expected to perform a conjugate addition across the highly electrophilic vinylogous amide moiety in pyrroledione 1 . The resulting enolate $\mathbf{9}$ is anticipated to undergo a proton transfer to produce less basic enolate 10, which is well suited for an intramolecular 5exo-trig cyclization affecting the ester function at C-5 and providing target structure $\mathbf{1 1}$ (Scheme 3).

To evaluate this idea we carried out the reaction of methyl 3benzoyl-4,5-dioxo-1-phenyl-4,5-dihydro- $1 \mathrm{H}$-pyrrole-2-carboxylate

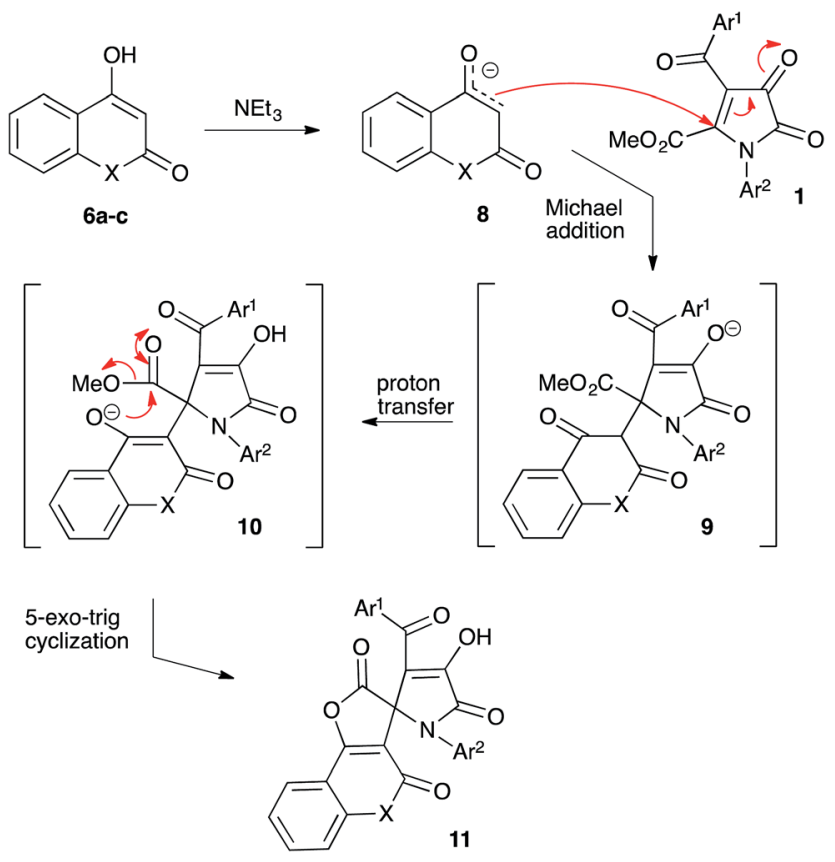

Scheme 3
Table 1 Spiro-condensation of 4-aroyl-5-methoxycarbonyl-1Hpyrrole-2,3-diones 1 with cyclic enoles 6

\begin{tabular}{|c|c|c|c|c|c|c|c|}
\hline \# & 1 & 6 & $\mathrm{X}$ & $\mathrm{Ar}^{1}$ & $\mathrm{Ar}^{2}$ & 11 & Yield $^{a}, \%$ \\
\hline 1 & $1 a$ & $6 a$ & $\mathrm{O}$ & $\mathrm{Ph}$ & $\mathrm{Ph}$ & $11 a a$ & 68 \\
\hline 2 & $1 b$ & $6 a$ & $\mathrm{O}$ & $4-\mathrm{MeOC}_{6} \mathrm{H}_{4}$ & $\mathrm{Ph}$ & $11 b a$ & 70 \\
\hline 3 & $1 c$ & $6 a$ & $\mathrm{O}$ & $\mathrm{Ph}$ & $4-\mathrm{MeOC}_{6} \mathrm{H}_{4}$ & 11ca & 84 \\
\hline 4 & $1 d$ & $6 a$ & $\mathrm{O}$ & $\mathrm{Ph}$ & $4-\mathrm{MeC}_{6} \mathrm{H}_{4}$ & 11da & 80 \\
\hline 5 & $1 e$ & $6 a$ & $\mathrm{O}$ & $4-\mathrm{MeC}_{6} \mathrm{H}_{4}$ & $\mathrm{Ph}$ & 11ea & 76 \\
\hline 6 & 1f & $6 a$ & $\mathrm{O}$ & $4-\mathrm{MeC}_{6} \mathrm{H}_{4}$ & $4-\mathrm{MeC}_{6} \mathrm{H}_{4}$ & $11 \mathrm{fa}$ & 78 \\
\hline 7 & $1 \mathrm{~g}$ & $6 a$ & $\mathrm{O}$ & $\mathrm{Ph}$ & $4-\mathrm{ClC}_{6} \mathrm{H}_{4}$ & 11ga & 67 \\
\hline 8 & $1 \mathrm{~h}$ & $6 a$ & $\mathrm{O}$ & $\mathrm{Ph}$ & $4-\mathrm{BrC}_{6} \mathrm{H}_{4}$ & 11ha & 76 \\
\hline 9 & $1 c$ & $6 b$ & $\mathrm{NMe}$ & $\mathrm{Ph}$ & $4-\mathrm{MeOC}_{6} \mathrm{H}_{4}$ & $11 \mathrm{cb}$ & 68 \\
\hline 10 & $1 d$ & $6 b$ & $\mathrm{NMe}$ & $\mathrm{Ph}$ & $4-\mathrm{MeC}_{6} \mathrm{H}_{4}$ & $11 d b$ & 69 \\
\hline 11 & $1 a$ & $6 c$ & NPh & $\mathrm{Ph}$ & $\mathrm{Ph}$ & $11 a c$ & 56 \\
\hline 12 & $1 g$ & $6 c$ & $\mathrm{NPh}$ & $\mathrm{Ph}$ & $4-\mathrm{ClC}_{6} \mathrm{H}_{4}$ & $11 \mathrm{gc}$ & 59 \\
\hline 13 & $1 \mathrm{i}$ & $6 c$ & NPh & $4-\mathrm{BrC}_{6} \mathrm{H}_{4}$ & $4-\mathrm{MeC}_{6} \mathrm{H}_{4}$ & 11ic & 67 \\
\hline
\end{tabular}

(1a, $\mathrm{Ar}^{1}=\mathrm{Ph}, \mathrm{Ar}^{2}=\mathrm{Ph}$ ) with 4-hydroxy-2H-chromen-2-one (6a). It was observed that an equimolar mixture of these starting materials when refluxed in toluene in the presence of catalytic amounts of triethylamine (10 mol\%) engaged in a quick reaction. The distinct purple color of pyrroledione faded away and the colorless product 11aa crystallized directly from the reaction mixture after cooling it down to $-10{ }^{\circ} \mathrm{C}$. This furo-coumarine was obtained as the sole product in good yield (68\%, Table 1 , entry 1). Reactions of other 4-aroyl-5-methoxycarbonyl pyrrolediones with $\mathbf{6 a}$ also proceeded uneventfully, affording the corresponding furo[3,2-c] coumarins very efficiently (Table 1 , entries 2-8). Reactions involving enolates generated from 4-hydroxy-2quinolones $\mathbf{6 b}$, $\mathbf{c}$ were carried out under the same conditions and also afforded the corresponding spiro furo[3,2-c] quinolones as sole products, albeit in somewhat lower yields (Table 1, entries 9-13). Formation of these structures was unambiguously confirmed by X-ray crystallography of compounds 11ba (Fig. 1) and 11db (Fig. 2).

We wondered, if the featured spiro-condensation would proceed selectively in more complex substrates, possibly

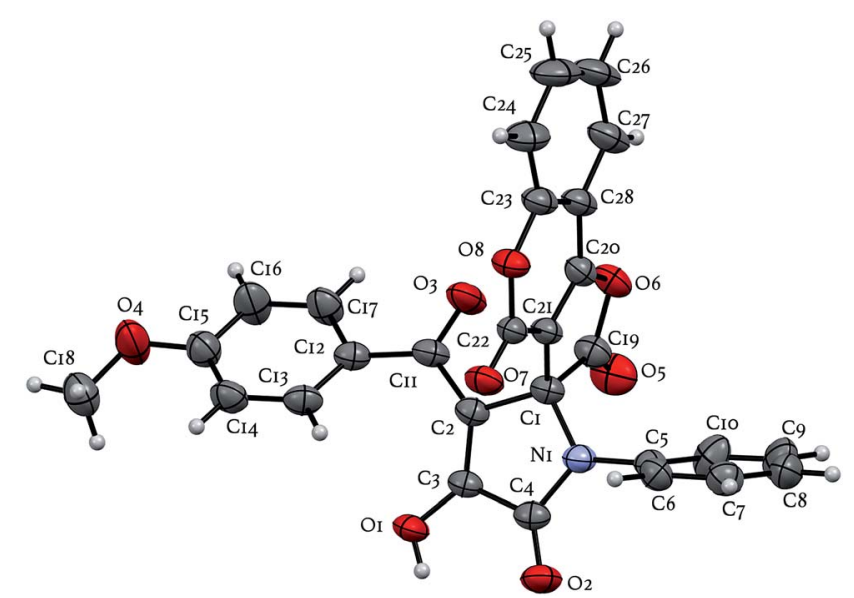

Fig. 1 ORTEP drawing of compound 11ba: showing 50\% probability amplitude displacement ellipsoids (CCDC \# 1486439†). 


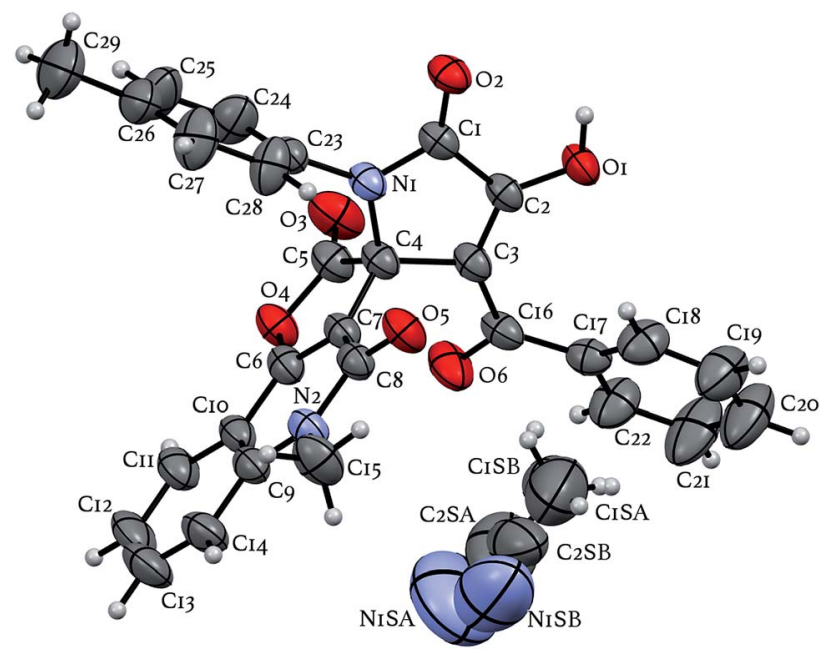

Fig. 2 ORTEP drawing of compound $11 \mathrm{db}$ : showing $50 \%$ probability amplitude displacement ellipsoids and a molecule of disordered crystallized solvent (acetonitrile) (CCDC \# 1486440†).
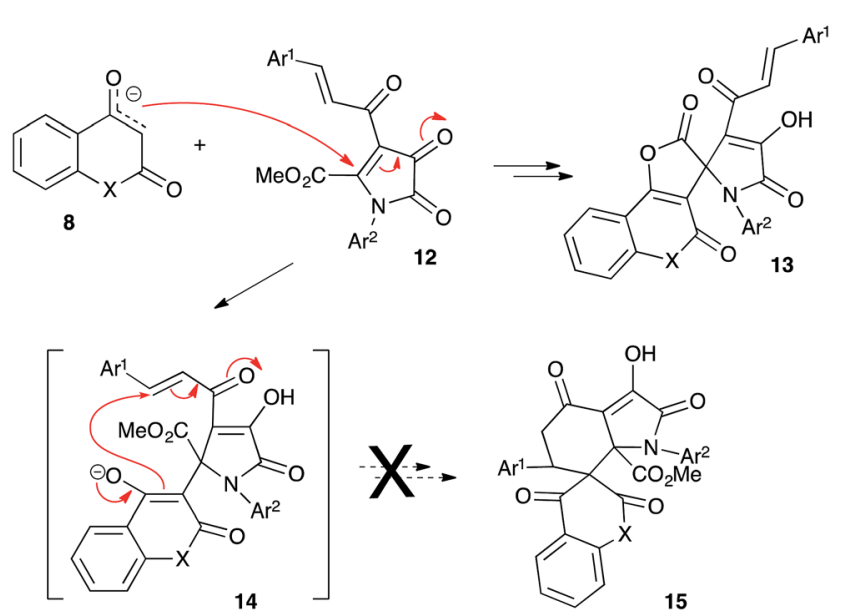

Scheme 4

allowing for alternative reaction pathways. For example, reaction of pyrrolediones 12 bearing a cinnamoyl substituent at C-4, with bis-nucleophilic enolates should proceed through intermediate 14, which could in principle undergo a second-fold enolexo-6-endo-trig Michael addition reaction to furnish different spiro scaffold 15 (Scheme 4). In our recent report we disclosed that in reactions of 4-cinnamoyl-pyrrole-2,3-diones 12 with five-membered cyclic enolates this process was prevalent. ${ }^{\mathbf{8}}$ Interestingly, in the presence of six-membered enolates, generated from heterocyclic precursors $\mathbf{6 a}-\mathbf{c}$ the reaction did not take this alternative route at all, proceeding instead via a "normal" 5-exo-trig lactonization pathway to afford the corresponding furo[3,2-c] coumarins and furo[3,2-c]quinolones 13 as sole products (Scheme 4, Table 2).

Next, we investigated the reactivity of 4,5-dimethoxycarbonyl-1 $H$-pyrrole-2,3-diones 16. After initial Michael addition of cyclic enolate $\mathbf{8}$, these substrates should provide intermediate 18, which can undergo the subsequent
Table 2 Spiro-condensation of 4-cinnamoyl-5-methoxycarbonyl$1 H$-pyrrole-2,3-diones 12 with cyclic enoles 6

\begin{tabular}{llllllll}
\hline$\#$ & $\mathbf{1 2}$ & $\mathbf{6}$ & $\mathrm{X}$ & $\mathrm{Ar}^{1}$ & $\mathrm{Ar}^{2}$ & $\mathbf{1 3}$ & $\mathrm{Yield}^{a}, \%$ \\
\hline 1 & $\mathbf{1 2 a}$ & $\mathbf{6 a}$ & $\mathrm{O}$ & $\mathrm{Ph}$ & $4-\mathrm{MeC}_{6} \mathrm{H}_{4}$ & $\mathbf{1 3 a a}$ & 67 \\
2 & $\mathbf{1 2 a}$ & $\mathbf{6 b}$ & $\mathrm{NMe}$ & $\mathrm{Ph}$ & $4-\mathrm{MeC}_{6} \mathrm{H}_{4}$ & $\mathbf{1 3 a b}$ & 75 \\
3 & $\mathbf{1 2 b}$ & $\mathbf{6 b}$ & $\mathrm{NMe}$ & $\mathrm{Ph}$ & $4-\mathrm{MeOC}_{6} \mathrm{H}_{4}$ & $\mathbf{1 3 b b}$ & 78 \\
4 & $\mathbf{1 2 c}$ & $\mathbf{6 b}$ & $\mathrm{NMe}$ & $4-\mathrm{MeOC}_{6} \mathrm{H}_{4}$ & $4-\mathrm{MeOC}_{6} \mathrm{H}_{4}$ & $\mathbf{1 3 c b}$ & 77 \\
5 & $\mathbf{1 2 b}$ & $\mathbf{6 c}$ & $\mathrm{NPh}$ & $\mathrm{Ph}$ & $4-\mathrm{MeOC}_{6} \mathrm{H}_{4}$ & $\mathbf{1 3 b c}$ & 65 \\
6 & $\mathbf{1 2 d}$ & $\mathbf{6 c}$ & $\mathrm{NPh}$ & $4-\mathrm{MeOC}_{6} \mathrm{H}_{4}$ & $4-\mathrm{MeC}_{6} \mathrm{H}_{4}$ & $\mathbf{1 3 d c}$ & 62
\end{tabular}

${ }^{a}$ Isolated yields of purified compounds $\mathbf{1 3}$.
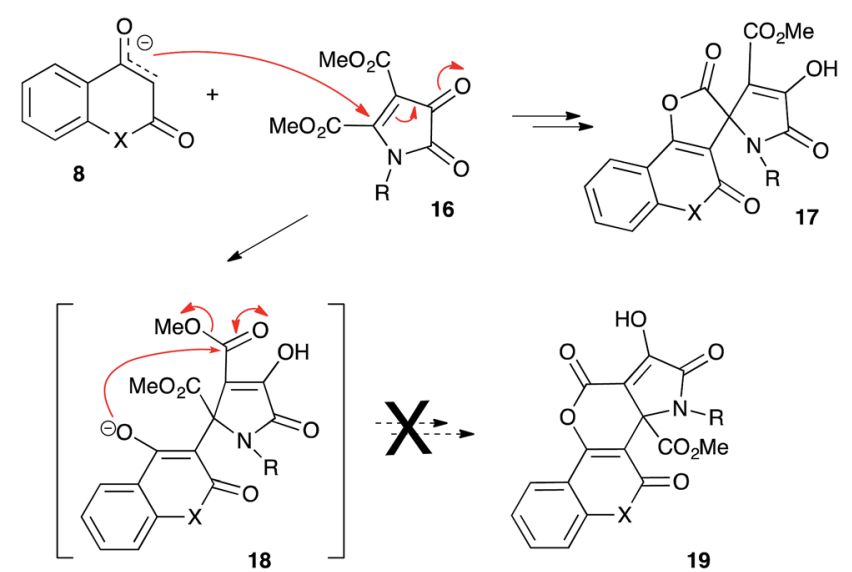

Scheme 5

Table 3 Spiro-condensation of 4,5-dimethoxycarbonyl-1H-pyrrole2,3-diones 16 with cyclic enoles 6

\begin{tabular}{lllllll}
\hline$\#$ & $\mathbf{1 6}$ & $\mathbf{6}$ & $\mathrm{X}$ & $\mathrm{Ar}^{1}$ & $\mathbf{1 7}$ & $\mathrm{Yield}^{a}, \%$ \\
\hline 1 & $\mathbf{1 6 a}$ & $\mathbf{6 a}$ & $\mathrm{O}$ & $\mathrm{CH}{ }_{2} \mathrm{Ph}$ & $\mathbf{1 7 a a}$ & 83 \\
2 & $\mathbf{1 6 b}$ & $\mathbf{6 a}$ & $\mathrm{O}$ & $\mathrm{Ph}$ & $\mathbf{1 7 b a}$ & 84 \\
3 & $\mathbf{1 6 c}$ & $\mathbf{6 a}$ & $\mathrm{O}$ & $4-\mathrm{MeC}_{6} \mathrm{H}_{4}$ & $\mathbf{1 7 c a}$ & 81 \\
4 & $\mathbf{1 6 a}$ & $\mathbf{6 c}$ & $\mathrm{NPh}$ & $\mathrm{CH}_{2} \mathrm{Ph}$ & $\mathbf{1 7 a c}$ & 79 \\
5 & $\mathbf{1 6 b}$ & $\mathbf{6 c}$ & $\mathrm{NPh}$ & $\mathrm{Ph}$ & $\mathbf{1 7 b c}$ & 69 \\
6 & $\mathbf{1 6 c}$ & $\mathbf{6 c}$ & $\mathrm{NPh}$ & $4-\mathrm{MeC}_{6} \mathrm{H}_{4}$ & $\mathbf{1 7 c c}$ & 64
\end{tabular}

${ }^{a}$ Isolated yields of purified compounds 17.

intramolecular nucleophilic attack involving one of the two available ester groups (Scheme 5). We were pleased to discover that these reactions also proceeded chemoselectively according to 5-exo-trig pathway, providing $\gamma$-lactones 17 as sole products in high yields (Table 3, entries 1-6). The alternative 6-exo-trig pathway was not realized at all, and the corresponding $\delta$-lactones 18 were not detected in the reaction mixtures (Scheme 5).

\section{Conclusions}

We have developed a novel cascade transformation combining an intermolecular conjugate addition of stabilized cyclic enolate (4- 
hydroxycoumarin or 4-hydroxy-2-quinolone) across a highly electrophilic vinylogous amide moiety of pyrrole-2,3-dione, and subsequent lactonization involving intramolecular nucleophilic attack of $O$-enolate at the ester substituent at C-5. The corresponding spiro furo[3,2-c]coumarins and furo[3,2-c] quinolones were formed as sole products in good yields. Remarkably, the introduction of competitive electrophilic substituents at C-4, such as cinnamoyl or methoxycarbonyl groups, did not divert the reaction from the described mechanistic route.

\section{Experimental part}

${ }^{1} \mathrm{H}$ and ${ }^{13} \mathrm{C}$ NMR spectra were recorded on a Bruker Avance-III spectrometer (400 or $100 \mathrm{MHz}$, respectively) equipped with BBO probe in $\mathrm{CDCl}_{3}$ or DMSO- $d_{6}$ using TMS as internal standard. IR spectra were recorded with a Perkin-Elmer Spectrum Two spectrometer from mulls in mineral oil. Melting points were measured with Stuart smp30 apparatus. X-ray crystallography was performed on Xcalibur Ruby diffractometer. The mass spectra were recorded on an Waters UPLC-MS instrument equipped with an ESI MS Xevo TQD detector. Elemental analyses were carried out on Vario MICRO Cube analyzer. Starting 5-methoxycarbonyl $1 \mathrm{H}$ pyrrole-2,3-diones 1a-i, 12a-d, and 16a-c were obtained as described in literature sources, ${ }^{9} \mathrm{~N}$-methyl- and $N$-phenyl-4hydroxy-2-quinolones $\mathbf{6 b}$, $\mathbf{c}$ were also prepared according to literature procedures. ${ }^{10}$ Anhydrous toluene was obtained by heating at reflux with molten sodium followed by distillation in under an atmosphere of dry nitrogen. Other reagents and solvents were purchased from commercial vendors and were used as received.

\section{3'-Benzoyl-4'-hydroxy-1'-(4-methoxyphenyl)-2H,4H-spiro[furo $[3,2-c]$ chromene-3, $2^{\prime}$-pyrrole $]-2,4,5^{\prime}\left(1^{\prime} H\right)$-trione (11ca, typical procedure)}

A solution of 4-hydroxycoumarin $6 \mathbf{6}(162 \mathrm{mg}, 1.00 \mathrm{mmol})$, methyl 3-benzoyl-1-(4-methoxyphenyl)-4,5-dioxo-4,5-dihydro- $1 H$-pyrrole2-carboxylate 1c (365 mg, $1.00 \mathrm{mmol})$, and $\mathrm{Et}_{3} \mathrm{~N}(10 \mathrm{mg}, 0.10$ $\mathrm{mmol})$ in anhydrous toluene $(5 \mathrm{~mL})$ was stirred at reflux for $1.5 \mathrm{~h}$ until purple color of pyrroledione faded away, and the solid precipitate formed. Then the reaction mixture was cooled to -10 ${ }^{\circ} \mathrm{C}$, and the resulted precipitate was filtered off, washed with hexane and recrystallized from toluene/chloroform $(2: 1)$ to afford 11ca (416 mg, 84\%) as a colorless crystals, mp $240-242{ }^{\circ} \mathrm{C}$ (dec.). ${ }^{1} \mathrm{H}$ NMR $\left(400 \mathrm{MHz}, \mathrm{CDCl}_{3}\right) \delta 7.88-7.85$ (m, 2H, Ar), 7.76$7.71(\mathrm{~m}, 1 \mathrm{H}, \mathrm{Ar}), 7.67-7.53(\mathrm{~m}, 2 \mathrm{H}, \mathrm{Ar}), 7.48-7.31$ (m, 4H, Ar), 7.19-7.13 (m, 2H, Ar), 6.87-6.83 (m, 2H, Ar), 3.74 (s, 3H, OMe); ${ }^{13} \mathrm{C}$ NMR $\left(100 \mathrm{MHz}, \mathrm{CDCl}_{3}\right) \delta 188.9,169.7,166.2,165.4,160.5$, 156.1, 155.9, 150.4 136.5, 134.9, 133.9, 129.6, 129.0, 128.6, 125.8, 125.1, 123.9, 117.7, 116.6, 115.5, 110.3, 100.9, 70.0, 55.6; IR ( NaCl, $\mathrm{cm}^{-1}$ ): 3408, 1848, 1736, 1707, 1676, 1652; MS: found 496.17; calcd for $\mathrm{C}_{28} \mathrm{H}_{18} \mathrm{NO}_{8}(\mathrm{M}+\mathrm{H})^{+}$496.10; EA: found $\mathrm{C} 67.84, \mathrm{H} 3.45, \mathrm{~N}$ 2.86; calcd for $\mathrm{C}_{28} \mathrm{H}_{17} \mathrm{NO}_{8}$ (495.44): C 67.88, H 3.46, N 2.83 .

3'-Benzoyl-4' -hydroxy-1'-phenyl-2H,4H-spiro[furo[3,2-c] chromene-3, $2^{\prime}$-pyrrole]-2,4,5' $\left(1^{\prime} H\right)$-trione (11aa)

Yield $316 \mathrm{mg}$ (68\%), mp 237-239 ${ }^{\circ} \mathrm{C}$ (dec.), colorless crystals. ${ }^{1} \mathrm{H} \mathrm{NMR}\left(400 \mathrm{MHz}, \mathrm{CDCl}_{3}\right.$ ) $\delta$ 7.89-7.85 (m, 2H, Ar), 7.73 (ddd, $J$
$=7.9,1.6,0.5 \mathrm{~Hz}, 1 \mathrm{H}, \mathrm{Ar}), 7.65-7.53(\mathrm{~m}, 2 \mathrm{H}, \mathrm{Ar}), 7.46-7.40(\mathrm{~m}$, 2H, Ar), 7.38-7.30 (m, 5H, Ar), 7.27-7.24 (m, 2H, Ar); ${ }^{13} \mathrm{C} \mathrm{NMR}$ $\left(100 \mathrm{MHz}, \mathrm{CDCl}_{3}\right) \delta 188.9,169.6,166.0,165.4,156.0,155.9$, 150.4 , 136.5, 134.9, 133.9, 133.5, 130.2, 129.8, 129.6, 128.6, 127.5, 125.1, 123.9, 117.7, 116.7, 110.3, 100.8, 69.7 ppm. IR $\left(\mathrm{NaCl}, \mathrm{cm}^{-1}\right): 3413,1851,1744,1711,1674,1648 ; \mathrm{MS}$ : found 466.15; calcd for $\mathrm{C}_{27} \mathrm{H}_{16} \mathrm{NO}_{7}(\mathrm{M}+\mathrm{H})^{+}$466.09; EA: found $\mathrm{C}$ 69.64, H 3.23, N 3.04; calcd for $\mathrm{C}_{27} \mathrm{H}_{15} \mathrm{NO}_{7}$ (465.42): C 69.68, H $3.25, \mathrm{~N} 3.01$.

\section{4'-Hydroxy-3' -(4-methoxybenzoyl)-1'-phenyl-2H,4H-spiro[furo} [3,2-c]chromene-3,2'-pyrrole]-2,4,5' (1'H)-trione (11ba)

Yield $347 \mathrm{mg}$ (70\%), mp $251-252{ }^{\circ} \mathrm{C}$ (dec.), colorless crystals. ${ }^{1} \mathrm{H}$ NMR (400 MHz, $\mathrm{CDCl}_{3}$ ) $\delta 7.92-7.87(\mathrm{~m}, 2 \mathrm{H}, \mathrm{Ar}), 7.78-7.71(\mathrm{~m}$, $1 \mathrm{H}, \mathrm{Ar}$ ), 7.77 (ddd, $J=8.4,7.5,1.6 \mathrm{~Hz}, 1 \mathrm{H}, \mathrm{Ar}), 7.41-7.23(\mathrm{~m}, 7 \mathrm{H}$, $\mathrm{Ar}), 6.97-6.92$ (m, 2H, Ar), 3.87 (s, 3H, OMe); ${ }^{13} \mathrm{C} \mathrm{NMR}(100 \mathrm{MHz}$, $\left.\mathrm{CDCl}_{3}\right) \delta 186.9,169.7,166.0,165.4,164.6,156.0,155.9,148.9$, $134.9,133.7,132.3,130.2,129.7,129.2,127.5,125.1,123.9$, $177.7,117.1,114.1,110.3,100.9,65.9,55.7 ; \mathrm{IR}\left(\mathrm{NaCl}, \mathrm{cm}^{-1}\right)$ : 3428, 1848, 1737, 1717, 1671, 1651; MS: found 496.19; calcd for $\mathrm{C}_{28} \mathrm{H}_{18} \mathrm{NO}_{8}(\mathrm{M}+\mathrm{H})^{+}$496.10; EA: found C 67.83, H 3.46, N 2.84; calcd for $\mathrm{C}_{28} \mathrm{H}_{17} \mathrm{NO}_{8}$ (495.44): C 67.88, H 3.46, N 2.83.

\section{3'-Benzoyl-4'-hydroxy-1' -(4-tolyl)-2H,4H-spiro[furo[3,2-c]} chromene-3,2'-pyrrole]-2,4,5' $\left(1^{\prime} H\right)$-trione (11da)

Yield $383 \mathrm{mg}(80 \%), \mathrm{mp} 203-205{ }^{\circ} \mathrm{C}$ (dec.), colorless crystals. ${ }^{1} \mathrm{H}$ NMR (400 MHz, $\left.\mathrm{CDCl}_{3}\right) \delta 7.92-7.87(\mathrm{~m}, 2 \mathrm{H}, \mathrm{Ar}), 7.78-7.71(\mathrm{~m}$, 1H, Ar), 7.68-7.53 (m, 2H, Ar), 7.48-7.40 (m, 2H, Ar), 7.37-7.30 (m, 2H, Ar), 7.19-7.06 (m, 4H, Ar), 2.21 (s, 3H, Me); ${ }^{13} \mathrm{C}$ NMR $\left(100 \mathrm{MHz}, \mathrm{CDCl}_{3}\right) \delta 188.9,169.7,166.1,165.4,156.0,155.9$, 150.5 , 140.1, 136.5, 134.9, 133.9, 130.8, 130.8, 129.6, 128.6, 127.3, 125.0, 123.9, 117.7, 116.6, 110.3, 100.9, 69.8, 21.3; IR $\left(\mathrm{NaCl}, \mathrm{cm}^{-1}\right): 3441,1851,1724,1708,1674,1646 ; \mathrm{MS}$ : found 480.19; calcd for $\mathrm{C}_{28} \mathrm{H}_{18} \mathrm{NO}_{7}(\mathrm{M}+\mathrm{H})^{+} 480.11$; EA: found $\mathrm{C} 70.12$, $\mathrm{H}$ 3.56, N 2.94; calcd for $\mathrm{C}_{28} \mathrm{H}_{17} \mathrm{NO}_{7}$ (479.44): C 70.15, H 3.57, N 2.92 .

\section{4'-Hydroxy-3'-(4-methylbenzoyl)-1'-phenyl-2H,4H-spiro[furo [3,2-c]chromene-3, $2^{\prime}$-pyrrole $]-2,4,5^{\prime}\left(1^{\prime} H\right)$-trione (11ea)}

Yield $364 \mathrm{mg}$ (76\%), mp $229-231{ }^{\circ} \mathrm{C}$ (dec.), colorless crystals. ${ }^{1} \mathrm{H}$ NMR (400 MHz, $\mathrm{CDCl}_{3}$ ) $\delta 7.81-7.71(\mathrm{~m}, 3 \mathrm{H}, \mathrm{Ar}), 7.63$ (ddd, $J=$ 8.4, 7.5, 1.6 Hz, 1H, Ar), 7.41-7.30 (m, 5H, Ar), 7.28-7.21 (m, $4 \mathrm{H}$, $\mathrm{Ar}), 2.40$ (s, 3H, Me); ${ }^{13} \mathrm{C}$ NMR (100 MHz, $\left.\mathrm{CDCl}_{3}\right) \delta$ 188.4, 169.7, $166.1,165.4$, 156.0, 155.9, 149.9, 145.2, 134.9, 133.8, 133.5, 130.2, 129.8, 129.8, 129.4, 127.5, 124.9, 123.9, 117.7, 117.0, 110.2, 100.7, 69.7, 22.0; IR ( $\left.\mathrm{NaCl}, \mathrm{cm}^{-1}\right): 3420,1854,1713,1673$, 1648; MS: found 480.18; calcd for $\mathrm{C}_{28} \mathrm{H}_{18} \mathrm{NO}_{7}(\mathrm{M}+\mathrm{H})^{+} 480.11$; EA: found C 70.11, H 3.55, N 2.92; calcd for $\mathrm{C}_{28} \mathrm{H}_{17} \mathrm{NO}_{7}$ (479.44): C 70.15, H 3.57, N 2.92 .

4'-Hydroxy-3' -(4-methylbenzoyl)-1' -(4-tolyl)-2H,4H-spiro[furo [3,2-c]chromene-3, $2^{\prime}$-pyrrole]-2,4,5' $\left(1^{\prime} H\right)$-trione (11fa)

Yield $385 \mathrm{mg}$ (78\%), mp $259-260{ }^{\circ} \mathrm{C}$ (dec.), colorless crystals. ${ }^{1} \mathrm{H}$ NMR $\left(400 \mathrm{MHz}, \mathrm{CDCl}_{3}\right) \delta 7.80-7.71(\mathrm{~m}, 3 \mathrm{H}, \mathrm{Ar}), 7.63$ (ddd, $J=$ 8.3, 7.5, 1.6 Hz, 1H, Ar), 7.38-7.29 (m, 2H, Ar), 7.27-7.22 (m, 3H, 
Ar), 7.17-7.11 (m, 3H, Ar), 2.40 (s, 3H, Me), 2.29 (s, 3H, Me); ${ }^{13} \mathrm{C}$ NMR (100 MHz, $\left.\mathrm{CDCl}_{3}\right) \delta$ 188.3, 169.8, 166.0, 165.3, 155.9, 149.8, $145.1,140.0,134.8,133.9,130.8,129.8,129.4$, 127.3, 125.0, 123.9, 117.7, 116.7, 110.4, 101.0, 69.8, 22.0, 21.3; IR (NaCl, $\left.\mathrm{cm}^{-1}\right)$ : 3438, 1848, 1723, 1673, 1646; MS: found 494.23; calcd for $\mathrm{C}_{29} \mathrm{H}_{20} \mathrm{NO}_{7}(\mathrm{M}+\mathrm{H})^{+}$494.12; EA: found $\mathrm{C} 70.54, \mathrm{H} 3.86, \mathrm{~N} 2.85$; calcd for $\mathrm{C}_{29} \mathrm{H}_{19} \mathrm{NO}_{7}$ (493.47): C 70.59, H 3.88, N 2.84.

\section{3'-Benzoyl-1' -(4-chlorophenyl)-4' -hydroxy- $2 \mathrm{H}, 4 \mathrm{H}$-spiro[furo} [3,2-c]chromene-3,2'-pyrrole]-2,4,5' $\left(1^{\prime} H\right)$-trione (11ga)

Yield $335 \mathrm{mg}$ (67\%), mp $233-235{ }^{\circ} \mathrm{C}$ (dec.), colorless crystals. ${ }^{1} \mathrm{H}$ NMR (400 MHz, $\mathrm{CDCl}_{3}$ ) $\delta 7.88-7.84(\mathrm{~m}, 2 \mathrm{H}, \mathrm{Ar}), 7.77$ (dd, $J=7.9$, $1.6 \mathrm{~Hz}, 1 \mathrm{H}, \mathrm{Ar}), 7.70-7.62(\mathrm{~m}, 1 \mathrm{H}, \mathrm{Ar}), 7.58$ (t, $J=7.4 \mathrm{~Hz}, 1 \mathrm{H}, \mathrm{Ar})$, $7.45(\mathrm{t}, J=7.7 \mathrm{~Hz}, 2 \mathrm{H}, \mathrm{Ar}), 7.40-7.30$ (m, 4H, Ar), 7.20 (dd, $J=$ 9.1, 2.4 Hz, 2H, Ar); $\left.{ }^{13} \mathrm{C} \mathrm{NMR} \mathrm{(100} \mathrm{MHz,} \mathrm{CDCl}_{3}\right) \delta$ 188.8, 169.6, 165.9, 165.6, 156.0 (2C), 150.0, 136.4, 136.0, 135.2, 134.1, 132.0, $130.5,129.6$, 128.9, 128.7, 125.2, 124.0, 117.8, 116.9, 110.2, 100.5, 69.6; IR (NaCl, $\left.\mathrm{cm}^{-1}\right): 3464,1849,1737,1709,1680,1656$; MS: found 500.17; calcd for $\mathrm{C}_{27} \mathrm{H}_{15} \mathrm{ClNO}_{7}(\mathrm{M}+\mathrm{H})^{+}$500.05; EA: found $\mathrm{C}$ 64.83, $\mathrm{H} \mathrm{2.80,} \mathrm{Cl} \mathrm{7.12,} \mathrm{N} \mathrm{2.81;} \mathrm{calcd} \mathrm{for} \mathrm{C}_{27} \mathrm{H}_{14} \mathrm{ClNO}_{7}$ (499.86): C 64.88, H 2.82, Cl 7.09, N 2.80.

\section{3'-Benzoyl-1' -(4-bromophenyl)-4' -hydroxy-2H,4H-spiro[furo [3,2-c]chromene-3,2'-pyrrole]-2,4,5' $\left(1^{\prime} H\right)$-trione (11ha)}

Yield $413 \mathrm{mg}$ (76\%), mp $235-236{ }^{\circ} \mathrm{C}$ (dec.), colorless crystals. ${ }^{1} \mathrm{H}$ NMR (400 MHz, $\mathrm{CDCl}_{3}$ ) $\delta 7.88-7.83(\mathrm{~m}, 2 \mathrm{H}, \mathrm{Ar}), 7.77$ (dd, $J=7.9$, $1.6 \mathrm{~Hz}, 1 \mathrm{H}, \mathrm{Ar}$ ), 7.66 (ddd, $J=8.9,7.5,1.6 \mathrm{~Hz}, 1 \mathrm{H}, \mathrm{Ar}$ ), 7.58 (ddd, $J=8.7,2.5,1.2 \mathrm{~Hz}, 1 \mathrm{H}, \mathrm{Ar}), 7.52-7.41$ (m, 4H, Ar), 7.39-7.33 (m, 2H, Ar), 7.16-7.11 (m, 2H, Ar); ${ }^{13} \mathrm{C}$ NMR (100 MHz, $\mathrm{CDCl}_{3}$ ) $\delta 188.8,169.6,165.8,165.6,156.0,156.0,149.9,136.4,135.2$, 134.1, 133.5, 132.6, 129.6, 129.1, 128.7, 125.2, 124.1, 124.0, 117.8, 116.9, 110.2, 100.5, 69.6; IR ( $\left.\mathrm{NaCl}, \mathrm{cm}^{-1}\right): 3473,1848$, 1738, 1709, 1678, 1654; MS: found 544.18; calcd for $\mathrm{C}_{27} \mathrm{H}_{15} \mathrm{BrNO}_{7}(\mathrm{M}+\mathrm{H})^{+}$544.00; EA: found $\mathrm{C} 59.54, \mathrm{H} 2.61, \mathrm{Br}$ 14.73, N 2.57; calcd for $\mathrm{C}_{27} \mathrm{H}_{14} \mathrm{BrNO}_{7}$ (544.31): C 59.58, H 2.59, Br 14.68, N 2.57.

3'-Benzoyl-4'-hydroxy-1'-(4-methoxyphenyl)-5-methyl-2H-spiro [furo[3,2-c]quinoline-3, $2^{\prime}$-pyrrole]-2,4,5' $\left(1^{\prime} H, 5 H\right)$-trione (11cb)

Yield $345 \mathrm{mg}$ (68\%), mp $243-244{ }^{\circ} \mathrm{C}$ (dec.), colorless crystals. ${ }^{1} \mathrm{H}$ NMR (400 MHz, $\mathrm{CDCl}_{3}$ ) $\delta 7.92-7.84(\mathrm{~m}, 3 \mathrm{H}, \mathrm{Ar}), 7.66$ (ddd, $J=$ 8.8, 7.2, 1.6 Hz, 1H, Ar), 7.56-7.47 (m, 1H, Ar), 7.42-7.28 (m, 4H, $\mathrm{Ar}), 7.22-7.14(\mathrm{~m}, 2 \mathrm{H}, \mathrm{Ar}), 6.85-6.80(\mathrm{~m}, 2 \mathrm{H}, \mathrm{Ar}), 3.74(\mathrm{~s}, 3 \mathrm{H}$, $\mathrm{Me}$ ), 3.63 (s, 3H, OMe); ${ }^{13} \mathrm{C}$ NMR (100 MHz, $\mathrm{CDCl}_{3}$ ) $\delta$ 188.9, 171.2 , 166.0, 161.0, 160.2, 157.7, 150.2, 141.9, 136.9, 133.5, 133.5, 129.7, 129.1, 128.5, 126.4, 124.2, 122.8, 116.6, 115.2, 115.1, 110.5, 105.6, 70.9, 55.5, 29.4; IR ( NaCl, $\left.\mathrm{cm}^{-1}\right): 3429,1838$, 1738, 1727, 1659, 1630; MS: found 509.21; calcd for $\mathrm{C}_{29} \mathrm{H}_{21} \mathrm{~N}_{2} \mathrm{O}_{7}$ $(\mathrm{M}+\mathrm{H})^{+}$509.13; EA: found $\mathrm{C} 68.44, \mathrm{H} \mathrm{3.93,} \mathrm{N} \mathrm{5.56;} \mathrm{calcd} \mathrm{for}$ $\mathrm{C}_{29} \mathrm{H}_{20} \mathrm{~N}_{2} \mathrm{O}_{7}$ (508.49): C 68.50, H 3.96, N 5.51.

3'-Benzoyl-4' -hydroxy-5-methyl-1' -(4-tolyl)-2H-spiro[furo[3,2-c] quinoline-3, 2'-pyrrole]-2,4,5' $\left(1^{\prime} \mathrm{H}, 5 \mathrm{H}\right)$-trione (11db)

Yield $339 \mathrm{mg}$ (69\%), mp 257-259 ${ }^{\circ} \mathrm{C}$ (dec.), colorless crystals. ${ }^{1} \mathrm{H}$ NMR (400 MHz, $\mathrm{CDCl}_{3}$ ) $\delta 7.92-7.82(\mathrm{~m}, 3 \mathrm{H}, \mathrm{Ar}), 7.68$ (ddd, $J=$
8.8, 7.2, 1.6 Hz, 1H, Ar), 7.54-7.49 (m, 1H, Ar), 7.40-7.26 (m, 4H, Ar), 7.18-7.09 (m, 4H, Ar), 3.62 (s, 3H, Me), 2.28 (s, 3H, $\left.\mathrm{C}_{6} \mathrm{H}_{4} \mathrm{Me}\right)$; ${ }^{13} \mathrm{C}$ NMR $\left(100 \mathrm{MHz}, \mathrm{DMSO}-d_{6}\right) \delta 188.5,171.7,165.6,159.2$, $156.6,153.8,141.0,138.7,136.6,133.6,133.1,131.4,130.2$, 128.9, 128.2, 126.6, 122.8 (2C), 116.0, 115.4, 108.9, 105.4, 69.4, 28.9, 20.5; IR (NaCl, $\mathrm{cm}^{-1}$ ): 3449, 1837, 1738, 1659, 1634; MS: found 493.21; calcd for $\mathrm{C}_{29} \mathrm{H}_{21} \mathrm{~N}_{2} \mathrm{O}_{6}(\mathrm{M}+\mathrm{H})^{+}$493.14; EA: found C 70.64, H 4.08, N 5.73; calcd for $\mathrm{C}_{29} \mathrm{H}_{20} \mathrm{~N}_{2} \mathrm{O}_{6}$ (492.49): C 70.73, H 4.09, N 5.69.

\section{3'-Benzoyl-4'-hydroxy-1',5-diphenyl-2H-spiro[furo[3,2-c]} quinoline-3, $2^{\prime}$-pyrrole]-2,4,5' $\left(1^{\prime} H, 5 H\right)$-trione (11ac)

Yield $303 \mathrm{mg}$ (56\%), mp 261-263 ${ }^{\circ} \mathrm{C}$ (dec.), colorless crystals. ${ }^{1} \mathrm{H}$ NMR (400 MHz, $\mathrm{CDCl}_{3}$ ) $\delta 7.89-7.81(\mathrm{~m}, 3 \mathrm{H}, \mathrm{Ar}), 7.62-7.46(\mathrm{~m}$, $4 \mathrm{H}, \mathrm{Ar}$ ), 7.45-7.28 (m, 8H, Ar), 7.23 (d, $J=7.2 \mathrm{~Hz}, 1 \mathrm{H}, \mathrm{Ar}), 7.15-$ 7.12 (m, 1H, Ar), 7.03 (d, $J=7.7 \mathrm{~Hz}, 1 \mathrm{H}, \mathrm{Ar}), 6.67$ (d, $J=8.6 \mathrm{~Hz}$, $1 \mathrm{H}, \mathrm{Ar}) ;{ }^{13} \mathrm{C} \mathrm{NMR}\left(100 \mathrm{MHz}, \mathrm{CDCl}_{3}\right) \delta$ 189.1, 171.1, 166.0, 161.6, $157.6,150.3,143.0,136.9,136.6$, 134.2, 133.6, 133.0, 130.4, 129.9, 129.6, 129.5, 129.4, 129.2, 128.8, 128.6, 127.6, 123.7, 123.0, 116.9, 110.2, 105.8, 70.6; IR $\left(\mathrm{NaCl}, \mathrm{cm}^{-1}\right)$ : 3443, 1835, 1732, 1718, 1637, 1626; MS: found 541.21; calcd for $\mathrm{C}_{33} \mathrm{H}_{21} \mathrm{~N}_{2} \mathrm{O}_{6}$ $(\mathrm{M}+\mathrm{H})^{+}$541.14; EA: found $\mathrm{C} 73.29, \mathrm{H} 3.71$, N 5.20; calcd for $\mathrm{C}_{33} \mathrm{H}_{20} \mathrm{~N}_{2} \mathrm{O}_{6}$ (540.53): C 73.33, H 3.73, N 5.18.

\section{3'-Benzoyl-1' -(4-chlorophenyl)-4'-hydroxy-5-phenyl-2H-spiro} [furo[3,2-c]quinoline-3, $2^{\prime}$-pyrrole]-2,4,5' $\left(1^{\prime} H, 5 H\right)$-trione (11gc)

Yield $339 \mathrm{mg}$ (59\%), mp 229-231 ${ }^{\circ} \mathrm{C}$ (dec.), colorless crystals. ${ }^{1} \mathrm{H}$ NMR (400 MHz, $\left.\mathrm{CDCl}_{3}\right) \delta 7.90-7.84(\mathrm{~m}, 3 \mathrm{H}, \mathrm{Ar}), 7.62-7.48(\mathrm{~m}$, $4 \mathrm{H}, \mathrm{Ar}$ ), 7.42 (ddd, $J=7.3,4.7,1.0 \mathrm{~Hz}, 3 \mathrm{H}, \mathrm{Ar}), 7.37-7.22(\mathrm{~m}, 5 \mathrm{H}$, Ar), 7.16-7.10 (m, 1H, Ar), 7.07-7.01 (m, 1H, Ar), 6.69 (d, $J=8.5$ $\mathrm{Hz}, 1 \mathrm{H}, \mathrm{Ar}) ;{ }^{13} \mathrm{C} \mathrm{NMR}\left(100 \mathrm{MHz}, \mathrm{CDCl}_{3}\right) \delta 189.0,170.9,165.9$, $161.7,157.6,149.8,143.0,136.8$, 136.5, 135.6, 133.8, 133.2, $132.7,130.4$, 130.2, 129.6, 129.5, 129.2, 129.0, 128.8, 128.6, 123.8, 123.2, 117.0, 116.8, 110.2, 105.3, 70.6; IR ( $\left.\mathrm{NaCl}, \mathrm{cm}^{-1}\right)$ : 3400, 1841, 1734, 1651, 1622; MS: found 575.23; calcd for $\mathrm{C}_{33} \mathrm{H}_{20} \mathrm{ClN}_{2} \mathrm{O}_{6}(\mathrm{M}+\mathrm{H})^{+}$575.10; EA: found $\mathrm{C} \mathrm{68.89,} \mathrm{H} \mathrm{3.46,} \mathrm{Cl}$ 6.11, N 4.80; calcd for $\mathrm{C}_{33} \mathrm{H}_{19} \mathrm{ClN}_{2} \mathrm{O}_{6}$ (574.97): $\mathrm{C} 68.94, \mathrm{H} 3.33, \mathrm{Cl}$ $6.17, \mathrm{~N} 4.87$.

3'-(4-Bromobenzoyl)-4' -hydroxy-5-phenyl-1' -(4-tolyl)-2H-spiro [furo[3,2-c]quinoline-3,2' -pyrrole]-2,4,5' $\left(1^{\prime} H, 5 H\right)$-trione (11ic)

Yield $362 \mathrm{mg}$ (67\%), mp 272-274 ${ }^{\circ} \mathrm{C}$ (dec.), colorless crystals. ${ }^{1} \mathrm{H} \mathrm{NMR}\left(400 \mathrm{MHz}, \mathrm{CDCl}_{3}\right) \delta 7.85(\mathrm{~d}, J=8.0 \mathrm{~Hz}, 1 \mathrm{H}, \mathrm{Ar}), 7.76$ $(\mathrm{d}, J=8.3 \mathrm{~Hz}, 2 \mathrm{H}, \mathrm{Ar}), 7.60-7.47(\mathrm{~m}, 3 \mathrm{H}, \mathrm{Ar}), 7.42-7.36(\mathrm{~m}$, $3 \mathrm{H}, \mathrm{Ar}$ ), 7.28-7.11 (m, 6H, Ar), 7.05 (d, J=7.0 Hz, 1H, Ar), 6.69 $(\mathrm{d}, J=8.7 \mathrm{~Hz}, 1 \mathrm{H}, \mathrm{Ar}), 2.33$ (s, 3H, Me); ${ }^{13} \mathrm{C} \mathrm{NMR}(100 \mathrm{MHz}$, $\left.\mathrm{CDCl}_{3}\right) \delta 188.2,177.1,165.7,161.7,157.6,151.1,142.9,139.8$, $136.5,135.8,133.0,131.8,131.3,130.7,130.4$, 130.4, 129.9, $129.5,129.3,128.8,128.5,127.8,123.7,123.1,120.1,117.1$, 116.3, 110.3, 105.8, 70.5, 21.4; IR ( $\left.\mathrm{NaCl}, \mathrm{cm}^{-1}\right): 3449,1836$, 1735, 1660, 1616; MS: found 633.15; calcd for $\mathrm{C}_{34} \mathrm{H}_{21} \mathrm{BrN}_{2} \mathrm{O}_{6}$ $(\mathrm{M}+\mathrm{H})^{+}$633.07; EA: found $\mathrm{C}$ 64.36, $\mathrm{H} 3.39, \mathrm{Br} 12.43, \mathrm{~N} 4.37$; calcd for $\mathrm{C}_{34} \mathrm{H}_{21} \mathrm{BrN}_{2} \mathrm{O}_{6}$ (633.45): $\mathrm{C}$ 64.47, $\mathrm{H}$ 3.34, $\mathrm{Br} 12.61$, $\mathrm{N} 4.42$. 
3'-Cinnamoyl-4'-hydroxy-1'-(4-tolyl)-2H,4H-spiro[furo[3,2-c] chromene-3, $2^{\prime}$-pyrrole]-2,4, $5^{\prime}\left(1^{\prime} H\right)$-trione (13aa)

Yield $339 \mathrm{mg}(67 \%), \mathrm{mp} 236-237^{\circ} \mathrm{C}$ (dec.), colorless crystals. ${ }^{1} \mathrm{H}$ NMR (400 MHz, DMSO- $d_{6}$ ) $\delta 11.25$ (br. s, 1H, OH), 7.88-7.83 (m, $2 \mathrm{H}, \mathrm{Ar}+\mathrm{CH}=\mathrm{CH}), 7.75-7.57(\mathrm{~m}, 5 \mathrm{H}, \mathrm{Ar}+\mathrm{CH}=\mathrm{CH}), 7.55-7.39$ $(\mathrm{m}, 4 \mathrm{H}, \mathrm{Ar}+\mathrm{CH}=\mathrm{CH}), 7.25(\mathrm{~d}, J=7.3 \mathrm{~Hz}, 2 \mathrm{H}, \mathrm{Ar}+\mathrm{CH}=\mathrm{CH})$, $7.02(\mathrm{~d}, J=7.4 \mathrm{~Hz}, 2 \mathrm{H}, \mathrm{Ar}+\mathrm{CH}=\mathrm{CH}), 2.24(\mathrm{~s}, 3 \mathrm{H}, \mathrm{Me}) ;{ }^{13} \mathrm{C} \mathrm{NMR}$ $\left(100 \mathrm{MHz}, \mathrm{DMSO}-d_{6}\right) \delta 182.0,170.9,165.8,163.5,157.6,155.2$, $154.8,143.1$, 139.1, 135.4, 133.4, 131.2, 130.8, 130.6, 129.1, 128.5, 126.6, 125.6, 123.3, 117.5, 115.6, 109.3, 101.8, 67.9, 20.6; IR $\left(\mathrm{NaCl}, \mathrm{cm}^{-1}\right)$ : 3420, 1843, 1716, 1670, 1642; MS: found 506.25; calcd for $\mathrm{C}_{30} \mathrm{H}_{20} \mathrm{NO}_{7}(\mathrm{M}+\mathrm{H})^{+}$506.12; EA: found $\mathrm{C} 71.36$, $\mathrm{H}$ 3.73, N 2.79; calcd for $\mathrm{C}_{30} \mathrm{H}_{19} \mathrm{NO}_{7}$ (505.48): C 71.28, H 3.79, N 2.77.

\section{3'-Cinnamoyl-4'-hydroxy-5-methyl-1'-(4-tolyl)-2H-spiro[furo} [3,2-c]quinoline-3,2'-pyrrole]-2,4,5' $\left(1^{\prime} H, 5 H\right)$-trione (13ab)

Yield $389 \mathrm{mg}$ (75\%), mp $276-277^{\circ} \mathrm{C}$ (dec.), colorless crystals. ${ }^{1} \mathrm{H}$ NMR (400 MHz, DMSO- $\left.d_{6}\right) \delta 7.83-7.75(\mathrm{~m}, 2 \mathrm{H}, \mathrm{Ar}+\mathrm{CH}=\mathrm{CH})$, 7.67-7.60 (m, 5H, $\mathrm{Ar}+\mathrm{CH}=\mathrm{CH}), 7.49-7.34(\mathrm{~m}, 4 \mathrm{H}, \mathrm{Ar}+\mathrm{CH}=$ $\mathrm{CH}), 7.18(\mathrm{~d}, J=8.1 \mathrm{~Hz}, 2 \mathrm{H}, \mathrm{Ar}+\mathrm{CH}=\mathrm{CH}), 7.04-6.99(\mathrm{~m}, 2 \mathrm{H}, \mathrm{Ar}$ $+\mathrm{CH}=\mathrm{CH}), 3.59(\mathrm{~s}, 3 \mathrm{H}, \mathrm{Me}), 2.21\left(\mathrm{~s}, 3 \mathrm{H}, \mathrm{C}_{6} \mathrm{H}_{4} \mathrm{Me}\right) ;{ }^{13} \mathrm{C} \mathrm{NMR}$ $\left(100 \mathrm{MHz}, \mathrm{DMSO}-d_{6}\right) \delta 181.9,171.8,165.6,158.9,156.5,156.5$, $142.9,140.9,138.6,134.2,133.4,131.5,130.7,130.1,129.0$, 128.3, 126.5, 123.4, 122.8, 122.7, 116.4, 115.9, 109.0, 106.1, 68.7, 28.8, 20.4; IR ( $\left.\mathrm{NaCl}, \mathrm{cm}^{-1}\right)$ : 3437, 1841, 1728, 1667, 1639; MS: found 519.35; calcd for $\mathrm{C}_{31} \mathrm{H}_{23} \mathrm{~N}_{2} \mathrm{O}_{6}(\mathrm{M}+\mathrm{H})^{+}$519.16; EA: found C 71.76, $\mathrm{H}$ 4.21, $\mathrm{N}$ 5.43; calcd for $\mathrm{C}_{31} \mathrm{H}_{22} \mathrm{~N}_{2} \mathrm{O}_{6}$ (518.53): C 71.81, H 4.28, N 5.40.

3'-Cinnamoyl-4' -hydroxy-1' -(4-methoxyphenyl)-5-methyl-2Hspiro[furo[3,2-c] quinoline-3, $2^{\prime}$-pyrrole]-2,4,5 $5^{\prime}\left(1^{\prime} H, 5 H\right)$-trione (13bb)

Yield $417 \mathrm{mg}$ (78\%), mp $297-298{ }^{\circ} \mathrm{C}$ (dec.), colorless crystals. ${ }^{1} \mathrm{H}$ NMR (400 MHz, DMSO- $\left.d_{6}\right) \delta 7.83-7.75(\mathrm{~m}, 2 \mathrm{H}, \mathrm{Ar}+\mathrm{CH}=\mathrm{CH})$, 7.74-7.51 (m, 5H, $\mathrm{Ar}+\mathrm{CH}=\mathrm{CH}), 7.49-7.36(\mathrm{~m}, 4 \mathrm{H}, \mathrm{Ar}+\mathrm{CH}=$ $\mathrm{CH})$, 7.07-7.00 (m, 2H, $\mathrm{Ar}+\mathrm{CH}=\mathrm{CH}), 6.97-6.92(\mathrm{~m}, 2 \mathrm{H}, \mathrm{Ar}+$ $\mathrm{CH}=\mathrm{CH}), 6.31$ (br. s, $1 \mathrm{H}, \mathrm{OH}), 3.68(\mathrm{~s}, 3 \mathrm{H}, \mathrm{OMe}), 3.60(\mathrm{~s}, 3 \mathrm{H}$, $\mathrm{Me}) ;{ }^{13} \mathrm{C}$ NMR $\left(100 \mathrm{MHz}, \mathrm{DMSO}-d_{6}\right) \delta 181.9,171.8,165.8,159.3$, $159.0,156.7$, 156.6, 142.9, 140.9, 134.2, 133.4, 130.7, 129.0, $128.3,126.4,123.4,122.8,122.7,116.4,116.0$, 114.9, 109.1, 106.1, 68.9, 55.2, 28.8; IR ( $\left.\mathrm{NaCl}, \mathrm{cm}^{-1}\right): 3456,1839,1728,1662$, 1639; MS: found 535.28; calcd for $\mathrm{C}_{31} \mathrm{H}_{23} \mathrm{~N}_{2} \mathrm{O}_{7}(\mathrm{M}+\mathrm{H})^{+}$535.15; EA: found $\mathrm{C} 69.78, \mathrm{H}$ 4.02, $\mathrm{N} 5.23$; calcd for $\mathrm{C}_{31} \mathrm{H}_{22} \mathrm{~N}_{2} \mathrm{O}_{7}$ (534.52): C 69.66, H 4.15, N 5.24.

4'-Hydroxy-1' -(4-methoxyphenyl)-3'-(3-(4-methoxyphenyl) acryloyl)-5-methyl-2H-spiro[furo[3,2-c] quinoline-3,2' -pyrrole]$2,4,5^{\prime}\left(1^{\prime} H, 5 H\right)$-trione $(13 \mathrm{cb})$

Yield $435 \mathrm{mg}$ (77\%), mp $281-282{ }^{\circ} \mathrm{C}$ (dec.), colorless crystals. ${ }^{1} \mathrm{H}$ NMR (400 MHz, DMSO- $\left.d_{6}\right) \delta 7.83-7.75(\mathrm{~m}, 2 \mathrm{H}, \mathrm{Ar}+\mathrm{CH}=\mathrm{CH})$, 7.68-7.48 (m, 5H, $\mathrm{Ar}+\mathrm{CH}=\mathrm{CH}), 7.42-7.38(\mathrm{~m}, 1 \mathrm{H}, \mathrm{Ar}+\mathrm{CH}=$ $\mathrm{CH}$ ), 7.08-6.98 (m, 4H, $\mathrm{Ar}+\mathrm{CH}=\mathrm{CH}), 6.97-6.92(\mathrm{~m}, 2 \mathrm{H}, \mathrm{Ar})$, 3.81 (s, 3H, OMe), 3.68 (s, 3H, OMe), 3.60 (s, 3H, Me); ${ }^{13} \mathrm{C}$ NMR $\left(100 \mathrm{MHz}, \mathrm{DMSO}-d_{6}\right) \delta 181.9,171.8,165.8,161.5,159.3,159.2$,
156.6, 155.9, 143.0, 140.9, 133.4, 130.3, 128.3, 126.8, 126.4, 122.8, 122.7, 120.9, 116.8, 115.9, 114.9, 114.6, 109.1, 106.1, 68.9, 55.3, 55.2, 28.8; IR (NaCl, $\mathrm{cm}^{-1}$ ): 3431, 1839, 1730, 1658, 1629; MS: found 565.27; calcd for $\mathrm{C}_{32} \mathrm{H}_{25} \mathrm{~N}_{2} \mathrm{O}_{8}(\mathrm{M}+\mathrm{H})^{+}$565.16; EA: found $\mathrm{C}$ 67.99, $\mathrm{H}$ 4.32, $\mathrm{N}$ 4.94; calcd for $\mathrm{C}_{32} \mathrm{H}_{24} \mathrm{~N}_{2} \mathrm{O}_{8}$ (564.55): $\mathrm{C}$ 68.08, H 4.29, N 4.96.

3'-Cinnamoyl-4' -hydroxy-1' -(4-methoxyphenyl)-5-phenyl-2Hspiro[furo[3,2-c] quinoline-3, $2^{\prime}$-pyrrole $]-2,4,5^{\prime}\left(1^{\prime} H, 5 H\right)$-trione (13bc)

Yield $388 \mathrm{mg}$ (65\%), mp 290-291 ${ }^{\circ} \mathrm{C}$ (dec.), colorless crystals. ${ }^{1} \mathrm{H} \mathrm{NMR}\left(400 \mathrm{MHz}, \mathrm{CDCl}_{3}\right) \delta 7.88(\mathrm{~d}, J=7.9 \mathrm{~Hz}, 1 \mathrm{H}, \mathrm{Ar}+\mathrm{CH}=$ $\mathrm{CH}), 7.75-7.26(\mathrm{~m}, 12 \mathrm{H}, \mathrm{Ar}+\mathrm{CH}=\mathrm{CH}), 7.25-7.21(\mathrm{~m}, 3 \mathrm{H}, \mathrm{Ar}+$ $\mathrm{CH}=\mathrm{CH}), 7.07(\mathrm{~d}, J=7.4 \mathrm{~Hz}, 1 \mathrm{H}, \mathrm{Ar}+\mathrm{CH}=\mathrm{CH}), 6.84(\mathrm{~d}, J=8.9$ $\mathrm{Hz}, 2 \mathrm{H}, \mathrm{Ar}+\mathrm{CH}=\mathrm{CH}), 6.71(\mathrm{~d}, J=8.7 \mathrm{~Hz}, 1 \mathrm{H}, \mathrm{Ar}+\mathrm{CH}=\mathrm{CH})$, 3.75 (s, 3H, OMe); ${ }^{13} \mathrm{C} \mathrm{NMR}\left(100 \mathrm{MHz}, \mathrm{CDCl}_{3}\right) \delta 183.4,171.2$, $166.0,161.5,160.3,157.7,144.7,142.9,136.7,134.8,132.9$, 130.9, 130.4, 129.5, 129.3, 129.1, 129.1, 129.0, 128.9, 126.6, 123.7, 123.7, 123.1, 117.9, 117.0, 115.2, 110.4, 105.4, 70.1, 55.6; IR $\left(\mathrm{NaCl}, \mathrm{cm}^{-1}\right): 3423,1839,1716,1667,1639 ; \mathrm{MS}$ : found 597.28; calcd for $\mathrm{C}_{36} \mathrm{H}_{25} \mathrm{~N}_{2} \mathrm{O}_{7}(\mathrm{M}+\mathrm{H})^{+}$597.17; EA: found $\mathrm{C}$ 72.39, $\mathrm{H}$ 4.01, N 4.76; calcd for $\mathrm{C}_{36} \mathrm{H}_{24} \mathrm{~N}_{2} \mathrm{O}_{7}$ (596.60): $\mathrm{C} 72.48, \mathrm{H}$ $4.06, \mathrm{~N} 4.70$.

4'-Hydroxy-3' -(3-(4-methoxyphenyl)acryloyl)-5-phenyl-1' -(4tolyl)-2H-spiro[furo[3,2-c]quinoline-3, $2^{\prime}$-pyrrole]-2,4,5' $\mathbf{5}^{\prime} \mathbf{1}^{\prime} \mathrm{H}$, $5 H)$-trione (13dc)

Yield $440 \mathrm{mg}$ (62\%), mp $272-273{ }^{\circ} \mathrm{C}$ (dec.), colorless crystals. ${ }^{1} \mathrm{H}$ $\mathrm{NMR}\left(400 \mathrm{MHz}, \mathrm{CDCl}_{3}\right) \delta 7.88(\mathrm{dd}, J=8.0,1.3 \mathrm{~Hz}, 1 \mathrm{H}, \mathrm{Ar}+\mathrm{CH}=$ $\mathrm{CH})$, 7.72-7.36 (m, 8H, $\mathrm{Ar}+\mathrm{CH}=\mathrm{CH}), 7.29-7.09(\mathrm{~m}, 6 \mathrm{H}, \mathrm{Ar}+$ $\mathrm{CH}=\mathrm{CH}), 7.05(\mathrm{~d}, J=7.7 \mathrm{~Hz}, 1 \mathrm{H}, \mathrm{Ar}+\mathrm{CH}=\mathrm{CH}), 6.87(\mathrm{~d}, J=8.8$ $\mathrm{Hz}, 2 \mathrm{H}, \mathrm{Ar}+\mathrm{CH}=\mathrm{CH}), 6.70(\mathrm{~d}, J=8.6 \mathrm{~Hz}, 1 \mathrm{H}, \mathrm{Ar}+\mathrm{CH}=\mathrm{CH})$, 3.83 (s, 3H, OMe), 2.29 (s, 3H, Me); ${ }^{13} \mathrm{C} \mathrm{NMR} \mathrm{(100} \mathrm{MHz}, \mathrm{CDCl}_{3}$ ) $\delta 183.2,171.2,166.0,162.2,161.4,157.7,144.7,142.9,139.6$, $136.7,132.8,131.5,130.8,130.5,130.4,130.4,129.4,129.3$, $128.9,127.5,127.4,123.7,123.0,121.3,117.0,114.7,110.4$, 106.5, 70.0, 55.6, 21.3; IR ( $\left.\mathrm{NaCl}, \mathrm{cm}^{-1}\right): 3443,1846,1737,1662$, 1637; MS: found 611.33; calcd for $\mathrm{C}_{37} \mathrm{H}_{27} \mathrm{~N}_{2} \mathrm{O}_{7}(\mathrm{M}+\mathrm{H})^{+}$611.18; EA: found $\mathrm{C} 72.71, \mathrm{H}$ 4.25, N 4.63; calcd for $\mathrm{C}_{37} \mathrm{H}_{26} \mathrm{~N}_{2} \mathrm{O}_{7}$ (610.62): C 72.78, H 4.29, N 4.59.

Methyl $1^{\prime}$-benzyl-4' -hydroxy-2,4,5' -trioxo-1 ${ }^{\prime}, 5^{\prime}$-dihydro- $2 H, 4 H$ spiro[furo[3,2-c] chromene-3, $2^{\prime}$-pyrrole]-3' -carboxylate (17aa)

Yield $359 \mathrm{mg}(83 \%), \mathrm{mp} 222-224{ }^{\circ} \mathrm{C}$ (dec.), colorless crystals. ${ }^{1} \mathrm{H}$ NMR (400 MHz, $\left.\mathrm{CDCl}_{3}\right) \delta 8.70$ (br. s, $\left.1 \mathrm{H}, \mathrm{OH}\right), 7.74-7.65(\mathrm{~m}, 2 \mathrm{H}$, Ar), 7.45-7.33 (m, 2H, Ar), 7.14-7.06 (m, 5H, Ar), 4.49 (dd, $J=$ 4.53, $\left.4.70 \mathrm{~Hz}, 2 \mathrm{H}, \mathrm{CH}_{2}\right), 3.72$ (s, 3H, OMe); ${ }^{13} \mathrm{C}$ NMR (100 MHz, $\left.\mathrm{CDCl}_{3}\right) \delta 168.9,164.1,164.0,163.0,157.8,155.9,155.1,135.0$, 133.0, 129.2, 128.8, 128.7, 125.1, 123.4, 117.7, 110.2, 108.2, 101.5, 65.6, 52.8, 46.0; IR ( $\left.\mathrm{NaCl}, \mathrm{cm}^{-1}\right): 3202,1843,1714,1678$, 1662, 1650; MS: found 434.15; calcd for $\mathrm{C}_{23} \mathrm{H}_{16} \mathrm{NO}_{8}(\mathrm{M}+\mathrm{H})^{+}$ 434.09; EA: found $\mathrm{C} 63.71, \mathrm{H} 3.46, \mathrm{~N} 3.28$; calcd for: $\mathrm{C}_{23} \mathrm{H}_{15} \mathrm{NO}_{8}$ (433.37): C 63.75, H 3.49, N 3.23. 
Methyl 4'-hydroxy-2,4,5' -trioxo-1'-phenyl-1', $5^{\prime}$-dihydro- $2 H, 4 H$ spiro[furo[3,2-c] chromene-3,2'-pyrrole]-3'-carboxylate (17ba)

Yield $352 \mathrm{mg}$ (84\%), mp $206-208{ }^{\circ} \mathrm{C}$ (dec.), colorless crystals. ${ }^{1} \mathrm{H}$ NMR (400 MHz, $\mathrm{CDCl}_{3}$ ) $\delta 8.62$ (br. s, $\left.1 \mathrm{H}, \mathrm{OH}\right), 7.72-7.65(\mathrm{~m}, 2 \mathrm{H}$, $\mathrm{Ar}), 7.43-7.27$ (m, 5H, Ar), 7.24-7.19 (m, 2H, Ar), $3.79(\mathrm{~s}, 3 \mathrm{H}$, $\mathrm{OMe}) ;{ }^{13} \mathrm{C}$ NMR $\left(100 \mathrm{MHz}, \mathrm{CDCl}_{3}\right) \delta 169.5,164.7,163.4,163.1$, $157.6,156.0$, 155.6, 135.1, 133.8, 130.2, 129.7, 127.6, 125.2, 123.6, 117.9, 110.1, 108.0, 101.1, 68.1, 52.9; IR ( $\left.\mathrm{NaCl}, \mathrm{cm}^{-1}\right)$ : 3279, 1837, 1738, 1716, 1645; MS: found 420.14; calcd for $\mathrm{C}_{22} \mathrm{H}_{14} \mathrm{NO}_{8}(\mathrm{M}+\mathrm{H})^{+}$420.07; EA: found C 62.91, H 3.06, N 3.38; calcd for $\mathrm{C}_{22} \mathrm{H}_{13} \mathrm{NO}_{8}$ (419.35): C 63.01; H 3.12; N 3.34.

Methyl 4'-hydroxy-2,4,5'-trioxo-1'-(4-tolyl)-1', $5^{\prime}$-dihydro- $2 H, 4 H$ spiro[furo[3,2-c] chromene-3,2'-pyrrole]-3'-carboxylate (17ca)

Yield $351 \mathrm{mg}$ (81\%), mp $210-212{ }^{\circ} \mathrm{C}$ (dec.), colorless crystals. ${ }^{1} \mathrm{H}$ NMR (400 MHz, $\left.\mathrm{CDCl}_{3}\right) \delta 7.71-7.64(\mathrm{~m}, 2 \mathrm{H}, \mathrm{Ar}), 7.43-7.33(\mathrm{~m}$, 2H, Ar), 7.17-7.07 (m, 4H, Ar), 6.30 (wide s, 1H, OH), 3.77 (s, 3H, OMe), 2.27 (s, 3H, Me); ${ }^{13} \mathrm{C}$ NMR (100 MHz, $\mathrm{CDCl}_{3}$ ) $\delta$ 169.6, $164.7,163.7$, 163.1, 157.4, 156.0, 155.6, 140.0, 135.0, 130.9, 130.8, 127.4, 125.1, 123.7, 117.9, 110.1, 107.9, 101.2, 68.3, 52.8, 21.3; IR ( $\left.\mathrm{NaCl}, \mathrm{cm}^{-1}\right)$ : 3148, 1852, 1743, 1722, 1692, 1652; MS: found 434.18; calcd for $\mathrm{C}_{23} \mathrm{H}_{16} \mathrm{NO}_{8}(\mathrm{M}+\mathrm{H})^{+}$434.09; EA: found $\mathrm{C}$ 63.71, H 3.46, N 3.28; calcd for $\mathrm{C}_{23} \mathrm{H}_{15} \mathrm{NO}_{8}$ (433.37): $\mathrm{C} 63.75, \mathrm{H}$ $3.49, \mathrm{~N} 3.23$.

Methyl 1'-benzyl-4' -hydroxy-2,4,5' -trioxo-5-phenyl-1' $, 4,5,5^{\prime}$ tetrahydro- $2 H$-spiro[furo[3,2-c] quinoline-3,2' carboxylate (17ac)

Yield $401 \mathrm{mg}$ (79\%), mp $244-246{ }^{\circ} \mathrm{C}$ (dec.), colorless crystals. ${ }^{1} \mathrm{H}$ NMR (400 MHz, $\mathrm{CDCl}_{3}$ ) $\delta 8.72$ (br. s, $1 \mathrm{H}, \mathrm{OH}$ ), 7.80 (dd, $J=8.0$, $1.4 \mathrm{~Hz}, 1 \mathrm{H}, \mathrm{Ar}), 7.62-7.44(\mathrm{~m}, 4 \mathrm{H}, \mathrm{Ar}), 7.31(\mathrm{td}, J=7.7,0.9 \mathrm{~Hz}$, $1 \mathrm{H}, \mathrm{Ar}$ ), 7.22-7.05 (m, 7H, Ar), 6.74 (d, J=8.6 Hz, 1H, Ar), 4.57 (s, $\left.2 \mathrm{H}, \mathrm{CH}_{2}\right), 3.70$ (s, 3H, OMe); ${ }^{13} \mathrm{C} \mathrm{NMR}\left(100 \mathrm{MHz}, \mathrm{CDCl}_{3}\right) \delta 170.3$, $164.3,163.2$, 160.9, 157.7, 157.2, 142.9, 136.5, 134.2, 133.1, $130.4,129.4,129.5,129.3,129.1,129.0,128.6,128.3,123.3$, 123.1, 117.0, 110.1, 108.6, 106.1, 66.6, 52.6, 45.9; IR ( NaCl, $\left.\mathrm{cm}^{-1}\right): 3448,1835,1732,1712,1697,1651,1632 ; \mathrm{MS}$ : found 509.28; calcd for $\mathrm{C}_{29} \mathrm{H}_{21} \mathrm{~N}_{2} \mathrm{O}_{7}(\mathrm{M}+\mathrm{H})^{+}$509.13; EA: found $\mathrm{C}$ 68.43, H 3.93, N 5.54; calcd for $\mathrm{C}_{29} \mathrm{H}_{20} \mathrm{~N}_{2} \mathrm{O}_{7}$ (508.49): C 68.50, H 3.96, N 5.51.

Methyl 4'-hydroxy-2,4,5' -trioxo-1 $^{\prime}, 5$-diphenyl-1' $, 4,5,5^{\prime}$ tetrahydro- $2 H$-spiro[furo[3,2-c] quinoline-3,2' -pyrrole $]-3^{\prime}$ carboxylate (17bc)

Yield $341 \mathrm{mg}$ (69\%), mp $255-256{ }^{\circ} \mathrm{C}$ (dec.), colorless crystals. ${ }^{1} \mathrm{H}$ NMR (400 MHz, $\mathrm{CDCl}_{3}$ ) $\delta 8.77$ (br. s, $1 \mathrm{H}, \mathrm{OH}$ ), 7.81 (ddd, $J=8.0$, 1.5, $0.5 \mathrm{~Hz}, 1 \mathrm{H}, \mathrm{Ar}$ ), 7.63-7.49 (m, 3H, Ar), 7.45 (ddd, $J=8.8,6.6$, $3.0 \mathrm{~Hz}, 1 \mathrm{H}, \mathrm{Ar}$ ), 7.36-7.23 (m, 7H, Ar), 7.03 (ddd, $J=6.4,2.9,1.5$ $\mathrm{Hz}, 1 \mathrm{H}, \mathrm{Ar}$ ), 6.72 (d, $J=7.3 \mathrm{~Hz}, 1 \mathrm{H}, \mathrm{Ar}), 3.77$ (s, 3H, OMe); ${ }^{13} \mathrm{C}$ NMR $\left(100 \mathrm{MHz}, \mathrm{CDCl}_{3}\right) \delta 170.9,163.7,163.3,161.2,157.6,157.4$, $143.0,136.5,134.0,133.2,130.5,130.5,129.8,129.5,129.3$, 129.2, 128.8, 127.7, 123.5, 123.2, 117.1, 110.0, 108.4, 105.9, 69.0, 52.7; IR ( $\mathrm{NaCl}, \mathrm{cm}^{-1}$ ): 3183, 1836, 1741, 1713, 1671, 1650, 1639; MS: found 495.22; calcd for $\mathrm{C}_{28} \mathrm{H}_{19} \mathrm{~N}_{2} \mathrm{O}_{7}(\mathrm{M}+\mathrm{H})^{+}$495.12; EA: found $\mathrm{C}$ 68.09, $\mathrm{H}$ 3.63, N 5.74; calcd for $\mathrm{C}_{28} \mathrm{H}_{18} \mathrm{~N}_{2} \mathrm{O}_{7}$ (494.46): C 68.02, H 3.67, N 5.67 .

Methyl 4'-hydroxy-2,4,5' -trioxo-5-phenyl-1' -(4-tolyl)-1' $, 4,5,5^{\prime}$ tetrahydro- $2 H$-spiro[furo[3,2-c]quinoline-3, $2^{\prime}$-pyrrole]- $3^{\prime}$ carboxylate (17cc)

Yield $325 \mathrm{mg}$ (64\%), mp $259-261{ }^{\circ} \mathrm{C}$ (dec.), colorless crystals. ${ }^{1} \mathrm{H}$ NMR (400 MHz, $\mathrm{CDCl}_{3}$ ) $\delta 8.74$ (wide s, $1 \mathrm{H}, \mathrm{OH}$ ), 7.81 (dd, $J=8.0$, $1.3 \mathrm{~Hz}, 1 \mathrm{H}, \mathrm{Ar}$ ), 7.65-7.49 (m, 3H, Ar), 7.45 (ddd, $J=8.7,7.2,1.6$ $\mathrm{Hz}, 1 \mathrm{H}, \mathrm{Ar}$ ), 7.34-7.22 (m, 2H, Ar), 7.18-7.01 (m, 5H, Ar), 6.74 (d, $J=8.6 \mathrm{~Hz}, 1 \mathrm{H}, \mathrm{Ar}), 3.76$ (s, 3H, OMe), 2.28 (s, 3H, Me); ${ }^{13} \mathrm{C} \mathrm{NMR}$ $\left(100 \mathrm{MHz}, \mathrm{CDCl}_{3}\right) \delta 170.9,163.8,163.3,161.1,157.7,157.5$, $143.0,139.5,136.6,133.1,131.6,130.5,130.5,129.6,129.2$, 128.8, 127.6, 123.5, 123.1, 117.1, 110.1, 108.3, 106.0, 69.0, 52.6, 21.3; IR: $\nu=3188,1840,1733,1708,1650,1639$; MS: found 509.25; calcd for $\mathrm{C}_{29} \mathrm{H}_{21} \mathrm{~N}_{2} \mathrm{O}_{7}(\mathrm{M}+\mathrm{H})^{+}$509.13; EA: found $\mathrm{C}$ 68.59, H 3.91, N 5.53; calcd for $\mathrm{C}_{29} \mathrm{H}_{20} \mathrm{~N}_{2} \mathrm{O}_{7}$ (508.49): C 68.50, H 3.96, N 5.51.

\section{Acknowledgements}

Financial support for this work was provided by Ministry of Education and Science of the Russian Federation (project \# 965).

\section{Notes and references}

1 (a) F. Medina, J. Marrero, M. Macias-Alonso, M. Gonzalez, I. Cordova-Guerrero, A. Garcia and S. Osegueda-Robles, Nat. Prod. Rep., 2015, 32, 1472; (b) L. Santana, E. Uriarte, F. Roleira, N. Milhazes and F. Borges, Curr. Med. Chem., 2004, 11, 3239; (c) N. Lopez-Gutierrez, R. Romero-Gonzalez, J. Vidal, A. Frenich, L. Santana, E. Uriarte, F. Roleira, N. Milhazes and F. Borges, Food Chem., 2016, 201, 59; (d) S. Ji, X. Qiao, Z. Li, Y. Wang, S. Yu, W. Liang, X. Lin and M. Ye, $R S C A d v$., 2015, 5, 45258; (e) S. Ji, W. Song, Y. Wang, W. Liang, K. Li, S. Tang, Q. Wang, X. Qiao, D. Zhou, S. Yu and M. Ye, J. Nat. Prod., 2016, 79, 281; (f) M. Neacsu, N. Vaughan, V. Raikos, S. Multari, G. Duncan, G. Duthie and W. Russell, Food Chem., 2015, 179, 159; (g) P. Pahari, U. Saikia, T. Das, C. Damodaran and J. Rohr, Tetrahedron, 2016, 72, 3324; (h) N. Raghav, S. Jangra, A. Kumar, S. Bhattacharyya, D. Wadhwa and J. Sindhu, RSC Adv., 2016, 6, 34588; (i) T. Won, I. Song, K. Kim, W. Yang, S. Lee, D. Oh, W. Oh, K. Oh and J. Shin, J. Nat. Prod., 2015, 78, 666; (j) G. Xi and Z. Liu, J. Agric. Food Chem., 2014, 62, 5636; (k) Y. Yuan, Y. Feng, F. Ren, S. Niu, X. Liu and Y. Che, Org. Lett., 2013, 15, 6050; (l) H. Morita, T. Dota and J. Kobayashi, Bioorg. Med. Chem. Lett., 2004, 14, 3665; (m) M. Xu and Y. S. Kim, Food Chem. Toxicol., 2014, 74, 311.

2 (a) S. Hajdok, J. Conrad, H. Leutbecher, S. Strobel, T. Schleid and U. Beifuss, J. Org. Chem., 2009, 74, 7230; (b) M. Rowley, J. Kulagowski, A. Watt, D. Rathbone, G. Stevenson, R. Carling, R. Baker, G. Marshall, J. Kemp, A. Foster, S. Grimwood, R. Hargreaves, C. Hurley, K. Saywell, M. Tricklebank and P. Leeson, J. Med. Chem., 1997, 40, 
4053; (c) Z. Xiao, N. Waters, C. Woodard, Z. Li and P. Lia, Bioorg. Med. Chem. Lett., 2001, 11, 2875; (d) S. Angeleska, P. Kefalas and A. Detsi, Tetrahedron Lett., 2013, 54, 2325; (e) F. O'Donnell, T. Smyth, V. Ramachandran and W. Smyth, Int. J. Antimicrob. Agents, 2010, 35, 30; $(f)$ K. Kawashima, T. Inoue, N. Tsutsumi and H. Endo, Biochem. Pharmacol., 1996, 51, 139; (g) C. Moulis, K. R. Wirasutisna, J. Gleye, P. Loiseau, E. Stanislas and C. Moretti, Phytochemistry, 1983, 22, 2095.

3 See, for example: (a) Y. Tsuda, S. Hosoi, N. Katagari, C. Kaneko and T. Sano, Chem. Pharm. Bull., 1993, 41, 2087; (b) Y. Tsuda, T. Ohshima, S. Hosoi, S. Kaneuchi, F. Kiuchi, T. Jun and T. Sano, Chem. Pharm. Bull., 1996, 44, 500.

4 (a) E. Ziegler, M. Eder, C. Belegratis and E. Prewedourakis, Monatsh. Chem., 1967, 98, 2249; (b) G. Kollenz, H. Igel and E. Ziegler, Monatsh. Chem., 1972, 103, 450; (c) B. Eistert, G. Muller and T. E. Arackal, Liebigs Ann. Chem., 1976, 1023; (d) T. Sano, J. Toda and Y. Tsuda, Chem. Pharm. Bull., 1983, 31, 356; (e) K. Isobe, C. Mohri, H. Sano, K. Mohri, H. Enomoto, T. Sano and Y. Tsuda, Chem. Pharm. Bull, 1989, 37, 3236; (f) B. Eistert, G. Miiller and T. Arackal, Liebigs Ann. Chem., 1976, 1031; $(g)$ L. George, P. Bernhardt, K. Netsch and C. Wentrup, Org. Biomol. Chem., 2004, 2, 3518; (h) H. Abd El-Nabi, Tetrahedron, 2002, 58, 135; (i) W. Fabian, J. Org. Chem., 2002, 67, 7475.
5 N. V. Bubnov, E. S. Denislamova, Z. G. Aliev and A. N. Maslivets, Russ. J. Org. Chem., 2011, 47, 526.

6 (a) Y. N. Bannikova, E. A. Sedegova, V. V. Khalturina and A. N. Maslivets, Russ. J. Org. Chem., 2007, 43, 1338; (b) E. S. Denislamova, A. Y. Dubovtsev, P. A. Slepukhin and A. N. Maslivets, Russ. J. Org. Chem., 2014, 50, 1017; A. Y. Dubovtsev, E. S. Denislamova, M. V. Dmiriev and A. N. Maslivets, Russ. J. Org. Chem., 2016, 52, 706.

7 (a) Y. N. Bannikova, A. N. Maslivets, Y. S. Rozhkova, Y. V. Shklyaev and Z. G. Aliev, Mendeleev Commun., 2005, 15, 158; (b) E. S. Denislamova and A. N. Maslivets, Russ. J. Org. Chem., 2010, 46, 389; (c) P. S. Silaichev, V. O. Filimonov, P. A. Slepukhin and A. N. Maslivets, Molecules, 2012, 17, 13787.

8 P. S. Silaichev, V. O. Filimonov, P. A. Slepukhin, M. Rubin and A. N. Maslivets, Eur. J. Org. Chem., 2015, 12, 2739.

9 (a) Y. Tsuda, Y. Horiguchi and T. Sano, Heterocycles, 1976, 7, 1237; (b) V. O. Filimonov, P. A. Slepukhin and A. N. Maslivets, Russ. J. Org. Chem., 2012, 48, 561; (c) K. Mohri, A. Kanie, Y. Horiguchi and K. Isobe, Heterocycles, 1999, 51, 2377.

10 (a) D. Buckle, B. Cantello, H. Smith and B. Spicer, J. Med. Chem., 1975, 7, 726; (b) E. Ziegler and K. Gelfert, Monatsh. Chem., 1959, 6, 822. 